\title{
Twenty-year longitudinal follow-up after liver transplantation: a single-center experience of 251 consecutive cases
}

\author{
Shin Hwang, Chul-Soo Ahn, Deok-Bog Moon, Tae-Yong Ha, Gi-Won Song, Dong-Hwan Jung, Gil-Chun Park, \\ Jung-Man Namgoong, Kyung Mo Kim, Sung-Gyu Lee
}

Department of Surgery, Asan Medical Center, University of Ulsan College of Medicine, Seoul, Korea

Background: The outcomes of liver transplantation (LT) has been improved, but the actual 20-year survival data are rarely presented.

Methods: A retrospective analysis of longitudinal follow-up data was performed to evaluate 20-year LT survivors. The institutional LT database was searched to identify patients who underwent primary LT during a 2-year period from January 2000 to December 2001. A study cohort of 251 patients was divided into three groups as adult living donor LT (LDLT; $n=207)$, adult deceased donor LT (DDLT; $n=22)$, and pediatric LT ( $n=22)$ groups.

Results: Common primary diseases were hepatitis B virus (HBV)-associated liver cirrhosis ( $\mathrm{n}=177,85.5 \%)$ and fulminant hepatic failure $(n=14,6.8 \%)$ in adult LDLT group; HBV-associated liver cirrhosis $(n=19,86.4 \%)$ in adult DDLT group; and biliary atresia ( $n=14$, $63.6 \%$ in pediatric LT group. The overall patient survival rates of adult LDLT cohort were $90.8 \%$ at 3 months, $86.0 \%$ at 1 year, $79.2 \%$ at 3 years, $77.3 \%$ at 5 years, $72.5 \%$ at 10 years, and $62.3 \%$ at 20 years, in which hepatocellular carcinoma recurrence, chronic rejection and de novo malignancy were the main cause of late patient death. The overall patient survival rates of adult DDLT cohort were $95.5 \%$ at 3 months, $86.4 \%$ at 1 year, $72.7 \%$ at 3 years, $72.7 \%$ at 5 years, $72.7 \%$ at 10 years, and $68.2 \%$ at 20 years. There was no statistical difference in overall patient survival rates between adult LDLT and DDLT groups ( $P=0.54)$. The overall patient survival rates of pediatric LT cohort were $95.5 \%$ at 3 months, $86.4 \%$ at 1 year, $86.4 \%$ at 3 years, $81.8 \%$ at 5 years, $81.8 \%$ at 10 years, and $77.3 \%$ at 20 years.

Conclusions: This is the first actual outcome of Korean single-center 20-year survival results of LT. It is necessary to analyze the causes of late graft failure in high-volume multicenter studies.

Corresponding author: Shin Hwang

E-mail: shwang@amc.seoul.kr

(C) The Korean Society for Transplantation

This is an Open Access article distributed under the terms of the Creative Commons Attribution Non-Commercial License (http://creativecommons.org/licenses/by-nc/4.0/) which permits unrestricted non-commercial use, distribution, and reproduction in any medium, provided the original work is properly cited. 